\title{
'Pseudo' treatment failure of pulmonary tuberculosis in association with a tuberculoma
}

\author{
Richard Long MD ${ }^{1}$, Sylvia Chomyc MLT BSc ${ }^{1}$, Evelina Der RN ${ }^{2}$, Daniel S Sitar $\mathrm{PhD}^{3}$ \\ ${ }^{1}$ Department of Medicine and Medical Microbiology, University of Alberta, Edmonton, Alberta; \\ ${ }^{2}$ Capital HealthTuberculosis Clinic, Edmonton, Alberta; ${ }^{3}$ Clinical Pharmacology Section, \\ University of Manitoba, Winnipeg, Manitoba
}

R Long, S Chomyc, E Der, DS Sitar. 'Pseudo' treatment failure of pulmonary tuberculosis in association with a tuberculoma. Can Respir J 2000;7(1):79-83.

Failure of tuberculosis patients to respond to treatment is usually explained by one or more of five mechanisms: improper drug prescription; patient nonadherence to prescribed therapy; primary or acquired drug resistance; drug malabsorption; and rarely, exogenous reinfection with a drug-resistant isolate. Response to treatment is best measured bacteriologically; two different smear and one culture criteria for failure are widely used. Patients meeting either smear, but not culture, criteria for treatment failure may be said to have 'pseudo' treatment failure. Whether a patient can meet both smear criteria for failure, and not have a mechanism for treatment failure nor meet culture criteria, is unknown. A case of 'pseudo' treatment failure is reported in which both smear criteria for failure were met, but no mechanism for failure was proven to be operative.
Pseudo-échec du traitement de la tuberculose pulmonaire associée à un tuberculome

RÉSUMÉ: L'échec du traitement de la tuberculose est en général causé par l'un des cinq phénomènes suivants : mauvais choix de médicament, non-observance au traitement de la part du patient, résistance primaire ou acquise au médicament, mauvaise absorption du médicament et, plus rarement, réinfection exogène par une souche résistante au médicament. La réponse au traitement se mesure le mieux par les analyses bactériologiques. On utilise le plus souvent deux frottis différents et une culture. Les patients qui répondent aux critères des frottis et non de la culture pour la définition de l'échec thérapeutique subiraient ce qu'on appelle un pseudo-échec du traitement. On ignore si un patient peut répondre aux critères associés au frottis pour l'échec thérapeutique et ne présenter aucun des phénomènes explicatifs ni répondre au critère associé à la culture. Un cas de pseudo-échec du traitement est décrit ici; il se caractérise par la présence des deux critères associés aux frottis, sans mécanisme explicatif.

Key Words: Treatment failure; Tuberculosis; Tuberculoma

Correspondence and reprints: Dr Richard Long, Department of Medicine, Division of Pulmonary Medicine, University of Alberta Hospitals, Room 2E4.21, Walter Mackenzie Centre, 8440-112 Street, Edmonton, Alberta T6G 2B7. Telephone 780-407-7551, fax 780-407-6384, e-mail richard.long@health.gov.ab.ca 


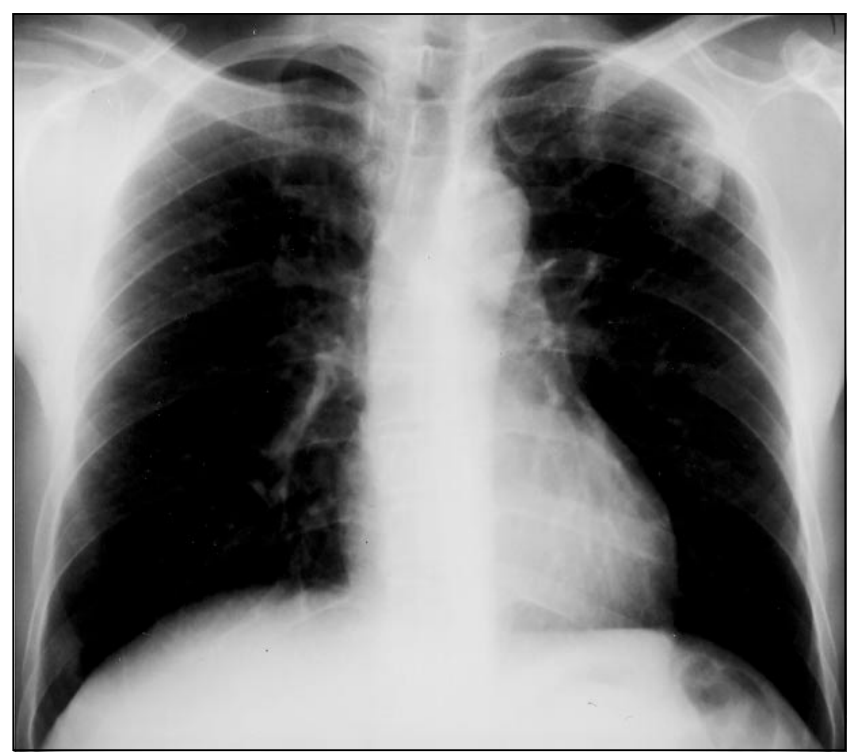

Figure 1) Posterior-anterior chest radiograph taken June 20, 1998, showing a $3.4 \mathrm{~cm}$ spherical lesion in the left upper lung zone and no disease elsewhere

A ssuming that a curative regimen is available and can be tolerated, instances of tuberculosis (TB) treatment failure are almost always explained by one or more of five mechanisms: improper drug prescription; patient nonadherence to prescribed therapy; primary or acquired drug resistance; drug malabsorption; and rarely, exogenous reinfection with a drugresistant isolate (1-3). Depending upon their availability, sputum smear (World Health Organization [WHO]) or culture (American Thoracic Society [ATS]) criteria may be used to determine the presence of treatment failure. For the WHO, treatment failure is said to exist when a patient remains or again becomes smear-positive five months or more after starting treatment. It also is a patient who was initially smear-negative before starting treatment and becomes smear-positive after the second month of treatment (4). For the ATS, failure exists when the sputum culture fails to convert after five to six months of treatment (5). Culture criteria are the gold standard, but they have limited application globally because most regions lack culture capability and rely totally upon direct microscopy to determine bacteriological response to treatment. When both smears and cultures are regularly available, and patients meet one or the other smear, but not culture, criteria for failure, they may be said to have 'pseudo' treatment failure. Whether a patient can meet both smear criteria for failure and not have a mechanism for treatment failure nor meet culture criteria is unknown. The following case demonstrates that, within the setting of a tuberculoma, such a response is indeed possible.

\section{CASE PRESENTATION}

A 45-year-old male immigrant to Canada from China presented on July 17, 1998 with a history of fever and cough of several weeks duration. He had no past history of TB nor any underlying condition known to compromise the immune system. Human immunodeficiency virus serology was negative.

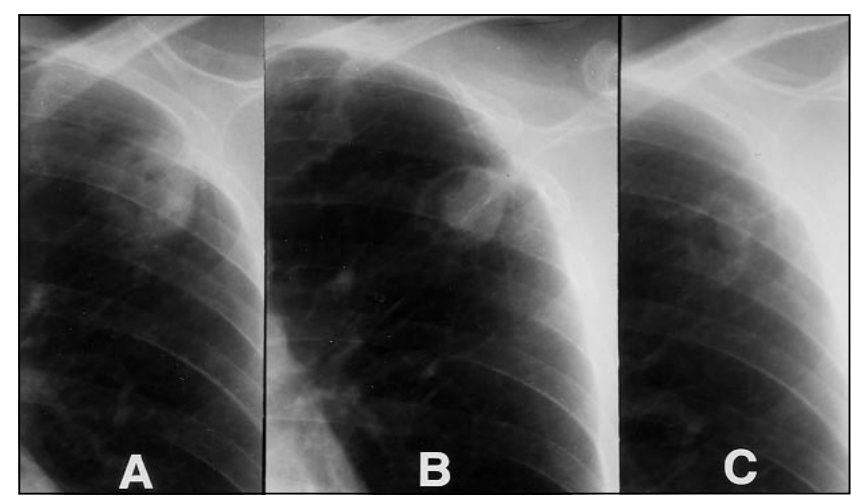

Figure 2) A Posterior-anterior view of the left upper hemithorax taken June 20,1998, showing a $3.4 \mathrm{~cm}$ spherical lesion in the left upper lung zone. An irregular area of translucency suggestive of cavitation is visible within the lesion. B Same view of the left hemithorax taken November 24, 1998, showing a reduction in the widest diameter of the lesion, now $3.0 \mathrm{~cm}$, with a well-defined, eccentric focus of cavitation. C Same view of the left hemithorax taken May 5, 1999, demonstrating further reduction in the diameter of the lesion, now $2.2 \mathrm{~cm}$, and further excavation

His cough was productive of white phlegm. He denied hemoptysis. A plain chest radiograph revealed a well-circumscribed, noncalcified spherical lesion measuring $3.4 \mathrm{~cm}$ in diameter in the apical-posterior segment of the left upper lobe (Figures 1,2A). An irregular area of translucency suggestive of cavitation was visible within this lesion. A sputum specimen that had been submitted by his referring physician on July 1, 1998 was acid-fast bacilli (AFB) smear negative (auraminerhodamine), but culture positive (BACTEC 460, BectonDickinson Microbiology Systems, Sparks, Maryland) for Mycobacterium tuberculosis, and was susceptible to all first-line antituberculous drugs (Provincial Laboratory of Public Health, Edmonton). Directly observed daily therapy was started on July 17 (isoniazid [INH] $300 \mathrm{mg}$, rifampin [RIF] $600 \mathrm{mg}$, pyrazinamide [PZA] $1500 \mathrm{mg}$ and ethambutol [EMB] $1000 \mathrm{mg}$ ) and continued until mid-September when it was reduced to twice weekly directly observed INH and RIF, $900 \mathrm{mg}$ and $600 \mathrm{mg}$, respectively. Complete adherence to the treatment regimen was observed, and no drug intolerance was reported or measured. Between the start of therapy and October 9, 1998, five additional sputum specimens were submitted; all were smear negative and three were culture positive (Figure 3). The patient's fever resolved, his weight increased from $58.3 \mathrm{~kg}$ on July 17 to $61.6 \mathrm{~kg}$ on October 13 , and a small $(3 \mathrm{~mm})$ reduction in the diameter of the presumed tuberculoma was reported on his chest radiograph. On October 13, a routine follow-up sputum specimen was AFB smear-positive in small numbers (less than 10 per slide).

This result, together with positive smears on subsequent sputum specimens, came as a complete surprise because the patient had been fully adherent to his directly observed therapy, had a fully drug susceptible isolate and had no gastrointestinal symptoms that would lead one to suspect drug malabsorption. A decision was made to admit the patient to 


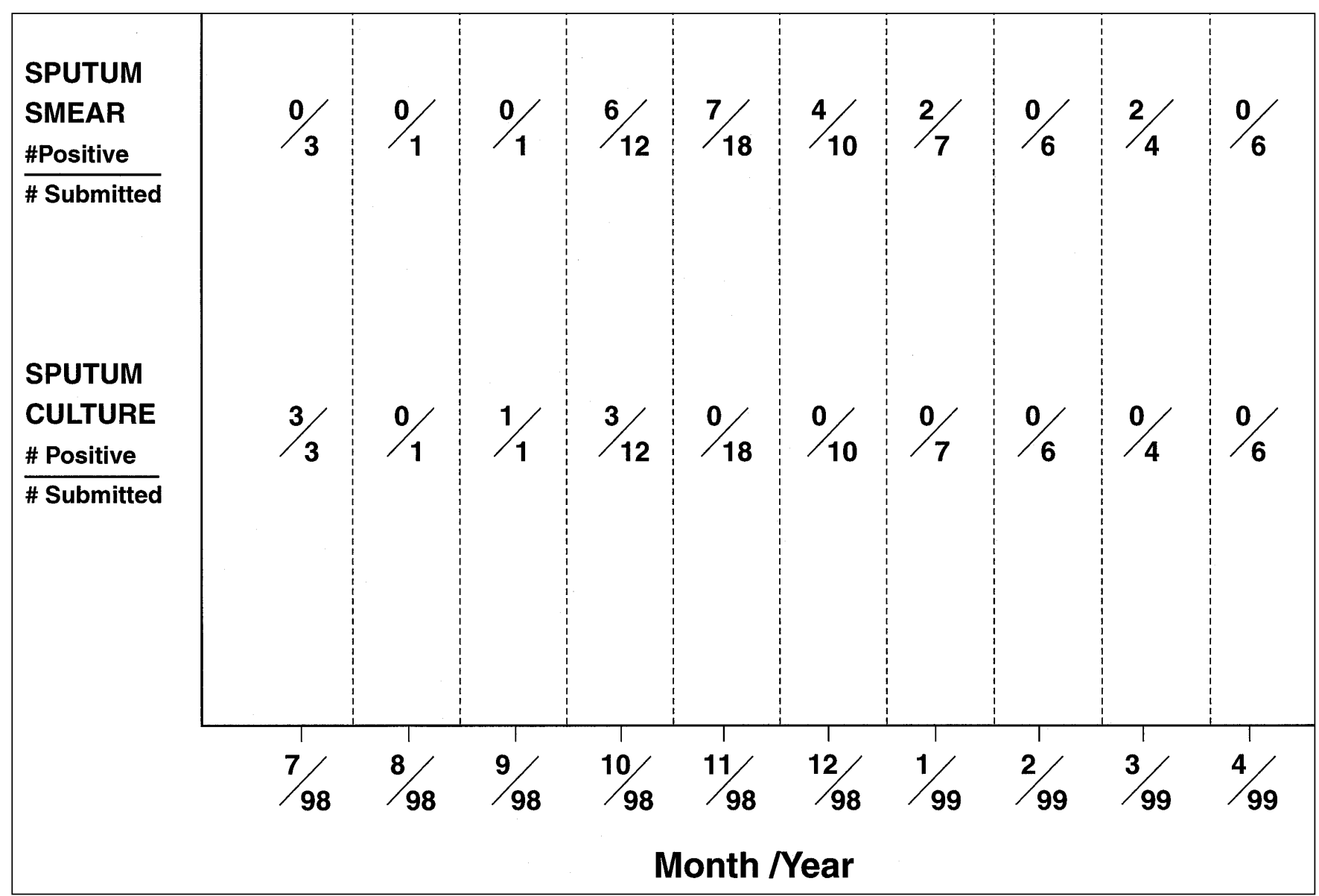

Figure 3) Sputum smear and culture results during the course of treatment of a 45-year-old patient. Results are expressed as the number positive over the number submitted each month

hospital for respiratory isolation, observation and further investigation. During this hospitalization, chest radiographs demonstrated reduction in size and increased excavation of the left upper lobe tuberculoma (Figure 2B). A computed tomographic scan of the thorax confirmed the presence of an eccentric focus of cavitation within the tuberculoma, as well as an eccentric quantity of calcification not visible on the plain film. Small volume hemoptysis was reported for the first time. On October 22, twice weekly intermittent therapy was discontinued and replaced with daily INH, RIF, PZA and EMB at the original dosages. Specimens of sputum from October 13, 20 and 24 were ultimately culture positive, and drug susceptibility testing was repeated on the isolate grown from the October 13 specimen; it was again fully drug susceptible. The original isolate from July 1 and the isolate from October 13 were also submitted to molecular diagnostics for molecular typing. The fingerprint profiles were generated by PVUII restriction digestion of chromosomal DNA followed by Southern blot hybridization with IS6110 as the probe (6). Both profiles were identical to each other. Finally, although no evidence of gastrointestinal disturbance was witnessed in or out of hospital, a noncompartmental pharmacokinetic analysis (10 plasma samples over $24 \mathrm{~h}$ ) of drug concentrations of INH (300 mg), RIF (600 mg) and PZA (1500 mg), measured after oral ingestion at 08:00 on November 25, was performed at steady state. The bioavailability of RIF and PZA was not different than reference norms (7). The bioavailability of INH was lower than the reference norm but as to what caused this no distinction could be made among altered absorption, altered distribution or altered first pass metabolism. This notwithstanding, during the entire sampling interval, plasma concentrations of INH were always well above the minimum inhibitory concentrations for $M$ tuberculosis $(0.1$ $\mathrm{mg} / \mathrm{L}$ or less) (8). Four-drug oral therapy was continued until December 8 when twice weekly INH and RIF was resumed.

Sputum specimens were taken on a regular basis (Figure 3), and surprisingly, smears continued to be positive into the eighth month of therapy; the last positive sputum smear was taken on March 15. Of a total of 55 specimens collected between October 13, 1998 and March 15, 1999, 20 (approximately 36\%) were smear positive by fluorescence microscopy, six specimens in moderate numbers (one to 10 per field at 200× magnification). Of 20 sputum specimens collected after Januray 17, 1999 (after six months of directly observed therapy), four (20\%) were smear-positive. Treatment was continued for a total of nine months; six months after the last positive culture (October 24, 1998). A plain chest radio- 
graph at the completion of treatment demonstrated considerable reduction in the size of the tuberculoma. Further excavation had occurred (Figure 2C).

\section{DISCUSSION}

We have described a patient who met both WHO criteria for tuberculosis treatment failure (4) (sputum smears remained positive beyond five months of treatment and initially smear-negative disease became smear-positive after the second month of treatment). However, by ATS (culture) criteria, the gold standard, he did not actually fail (5), nor did he have operative any mechanism known to cause treatment failure (1-3). The patient was prescribed an appropriate regimen of antituberculous drugs and was fully adherent to prescribed therapy; his isolate was and remained fully drug susceptible, and proof of drug malabsorption could not be established. In addition, molecular typing did not support the unlikely possibility that he was populated with more than one strain of $M$ tuberculosis, nor was he immunocompromized, although impairment of host immunological responses, such as occurs with human immunodeficiency virus infection, has little impact on bacteriological response to treatment (9).

It may be argued that our patient did not meet the second WHO criteria. Rather, his early negative smear results were simply a sampling artefact, because many more sputum specimens were submitted after sputum-smear positivity was detected than before positivity (Figure 3 ). However, the odds that none of six sputum specimens, submitted between July 1,1998 , the date the first specimen was submitted, and October 9, 1998, would be smear-positive are very low when six of $11(55 \%)$ sputum specimens submitted between October 13 and 31, 1998 were smear-positive. This is particularly true given that smear-positivity is more, not less likely, the closer a patient is to being untreated. The likelihood of a patient meeting the second WHO criteria and having 'pseudo' treatment failure is not known but is judged to be very small.

The development during treatment of smear-positive, culture-negative status is well recognized, and may cause an estimated $5 \%$ of sputum smear-positive pulmonary TB patients to meet the first WHO criteria for treatment failure (smear positive at five months) without actually failing (cultures negative) (10-12). The tendency during treatment for sputum cultures to become negative before sputum smears has been associated with advanced disease and with treatment regimens that contained rifampin (10). Fluorochrome staining may contribute to a persistently smear-positive state because the auramine-rhodamine stain is more sensitive than the Ziehl-Neelsen stain (13); organisms apparently dead or rendered noncultivable by chemotherapy may still be fluorescence positive. Consideration of the treatment response in our patient focused on the nature of his lung lesion.

Tuberculomas are isolated nodular masses caused by the tubercle bacillus; they range in size from 0.5 to $4 \mathrm{~cm}$ in diameter, and are smooth and sharply defined (14-18). Macleod and Smith (15) described the necrotic tissue that forms the core of most of these lesions as having three appearances: "the ghost of tuberculous granulation tissue" - an interlacing fibrous network enclosing poorly defined giant cells; "the ghost of bronchopneumonia" - where the alveolar structure of the lung is conserved; and completely amorphous areas without evidence of fibre structure or lining alveoli. Areas having this last appearance were reported as being sharply demarcated from areas of necrosis with a conserved structure. Amorphous necrotic tissue was generally seen well away from the edge of the lesion; where the tissue had been eccentrically placed and in relation to a bronchus, liquefaction and evacuation into the bronchus sometimes occurred. AFB were prominent in the amorphous areas. Conceivably, the unusual bacteriological response in our patient was because of the relative inability of antituberculous drugs to penetrate or destroy these isolated foci of tubercle bacilli, resulting in delayed liquefaction and erosion of the foci into the bronchus (19).

Reports of the finding of tubercle bacilli in the sputum of patients with tuberculomas have varied in different series; for example, Pugh and associates (17) reported the sputum positive for tubercle bacilli in only three of 28 cases, while Sochocky (18) reported sputum positive for tubercle bacilli on microscopy or on culture in 17 of 30 cases. Because closed tuberculosis lesions usually contain less than $10^{4}$ organisms (20), patients with isolated closed lesions would not be expected to be sputum smear-positive because the latter requires $10^{4}$ or more bacteria/mL of sputum to be present (21). The response to treatment of tuberculomas associated with bacillary positive sputum has not, to our knowledge, been reported previously. Given the importance of neoplasm in the differential diagnosis, tuberculomas are often resected (22), precluding any observation of their response to medical therapy alone.

In a recent report from Canada (12), 70\% of patients meeting the first WHO criteria for treatment failure did not actually have failure. As our patient demonstrates, it is possible not only to meet the first, but both WHO criteria for treatment failure without actually failing or having operative a mechanism for treatment failure. This might influence the management of tuberculomas in developing countries.

ACKNOWLEDGMENTS: The authors are very grateful to Dr Jim Barrie, for his review of the chest radiographs, and to Susan Falconer for her preparation of the manuscript. This work was supported by a University of Alberta Trust Account.

\section{REFERENCES}

1. Monitoring the patient. In: Maher D, Chaulet P,

Spinaci S, Harries A, eds. Treatment of Tuberculosis: Guidelines for National Programmes, 2nd edn. Geneva: World Health Organization, 1997:33-9.

2. Patel KB, Belmonte R, Crowe HM. Drug malabsorption and resistant tuberculosis in HIV infected patients. N Engl J Med 1995;332:336-7.

3. Small PM, Shafer RW, Hopewell PC, et al. Exogenous reinfection with multidrug-resistant Mycobacterium tuberculosis in patients with advanced HIV infection. N Engl J Med 1993;328:1137-44.

4. Case definitions. In: Maher D, Chaulet P, Spinaci S, Harries A, eds. Treatment of Tuberculosis: Guidelines for National Programmes, 2nd edn. Geneva: World Health Organization, 1997:18-24.

5. Bass JB Jr, Farer LS, Hopewell PC, et al. Treatment of tuberculosis and tuberculosis infection in adults and children. Am J Respir Crit Care Med 1994;149:1359-74.

6. Goyal M, Saunders NA, van Embden JDA, Young DB, Shaw RJ. 
Differentiation of Mycobacterium tuberculosis isolates by spoligotyping and IS6110 restriction fragment length polymorphism. J Clin Microbiol 1997;35:647-51.

7. Benet LZ, Oie S, Schwartz JB. Appendix II. Design and optimization of dosage regimens; pharmacokinetic data. In: Hardman JG, Limbiro LE, Molinoff PB, Ruddon RN, Gilman AG, eds. The Pharmacological Basis of Therapeutics, 9th edn. Toronto: McGraw Hill, 1996;1707-92.

8. Heifets LB. Drug susceptibility tests in the management of chemotherapy of tuberculosis. In: Heifets LB, ed. Drug susceptibility in the chemotherapy of mycobacterial infections. Boca Raton: CRC Press 1991:89-121.

9. Telzak EE, Fazal BA, Pollard CL, Turett GS, Justman JE, Blum S. Factors influencing time to sputum conversion among patients with smear positive pulmonary tuberculosis. Clin Infect Dis 1997;25:666-70.

10. Kim TC, Blackman RS, Heatwole KM, Kim T, Rochester DF. Acid-fast bacilli in sputum smears of patients with pulmonary tuberculosis. Am Rev Respir Dis 1984;129:264-8.

11. Vidal R, Martin-Casabona N, Juan A, Falgueras T, Miravitlles M. Incidence and significance of acid-fast bacilli in sputum smears at the end of antituberculous treatment. Chest 1996;109:1562-5.

12. Al-Moamary MS, Black W, Bessuille E, Elwood RK, Vedal S. The significance of the persistent presence of acid-fast bacilli in sputum smears in pulmonary tuberculosis. Chest 1999;116:726-31.
13. Gangadharam PR. The role of the laboratory in the management of tuberculosis patients. Semin Respir Med 1981;2:182-95.

14. Grenville-Mathers R. The natural history of so-called tuberculomas. J Thoracic Surg 1952;23:251-2.

15. MacLeod WM, Smith AT. Some observations on the historical appreciation, pathological development, and behavior of round tuberculous foci. Thorax 1952;7:334-53.

16. Hillerdal O. Tuberculoma of the lung. Acta Tuberc Scand 1954:34(Suppl):1-191.

17. Pugh DL, Jones ER, Martin WJ. 'Tuberculoma' of the Lung. Tubercle 1952;33:184-8

18. Sochocky S. Tuberculoma of the lung. Am Rev Tuberc 1958;78:403-10.

19. Wallis RS, Patil S, Cheon SH, et al. Drug tolerance in Mycobacterium tuberculosis. Antimicrob Agents Chemother 1999;43:2600-6.

20. Canetti G. Present aspects of bacterial resistance in tuberculosis. Am Rev Respir Dis 1965;92:687-703.

21. Yeager H Jr, Lacy J, Smith LR, LeMaister CA. Quantitative studies of mycobacterial populations in sputum and saliva. Am Rev Respir Dis 1966:95:998-1004.

22. Ishida T, Yokoyama H, Kaneito S, Sugid K, Sugimachi K, Hara N. Pulmonary tuberculoma and indications for surgery: radiographic and clinicopathological analysis. Respir Med 1992;86:431-6. 


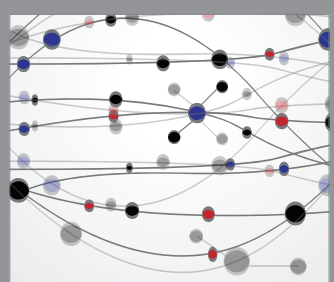

The Scientific World Journal
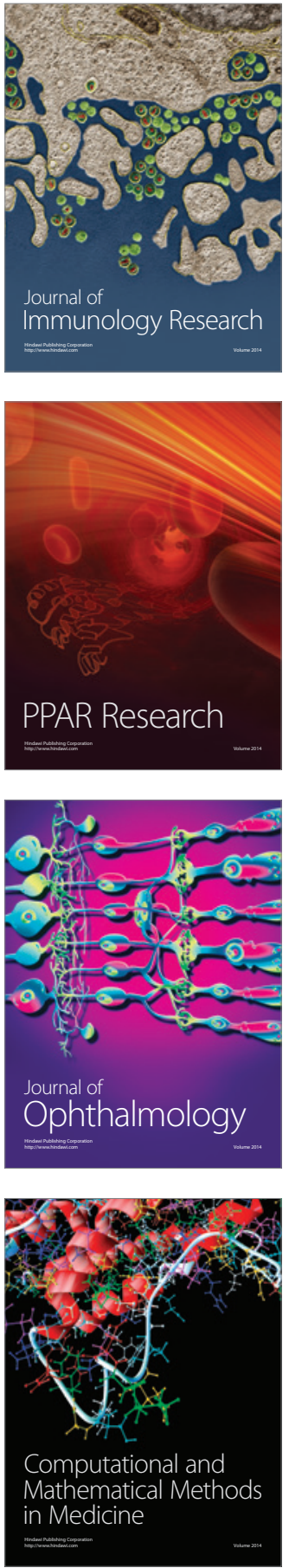

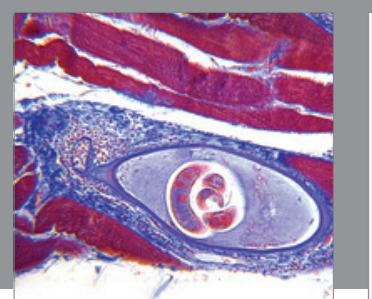

Gastroenterology Research and Practice

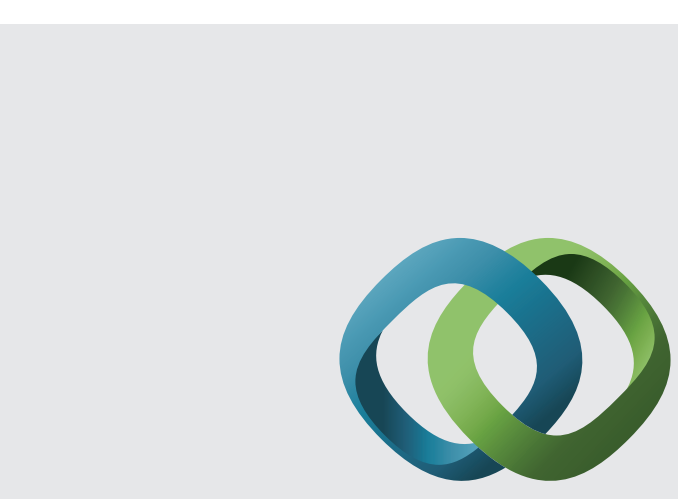

\section{Hindawi}

Submit your manuscripts at

http://www.hindawi.com
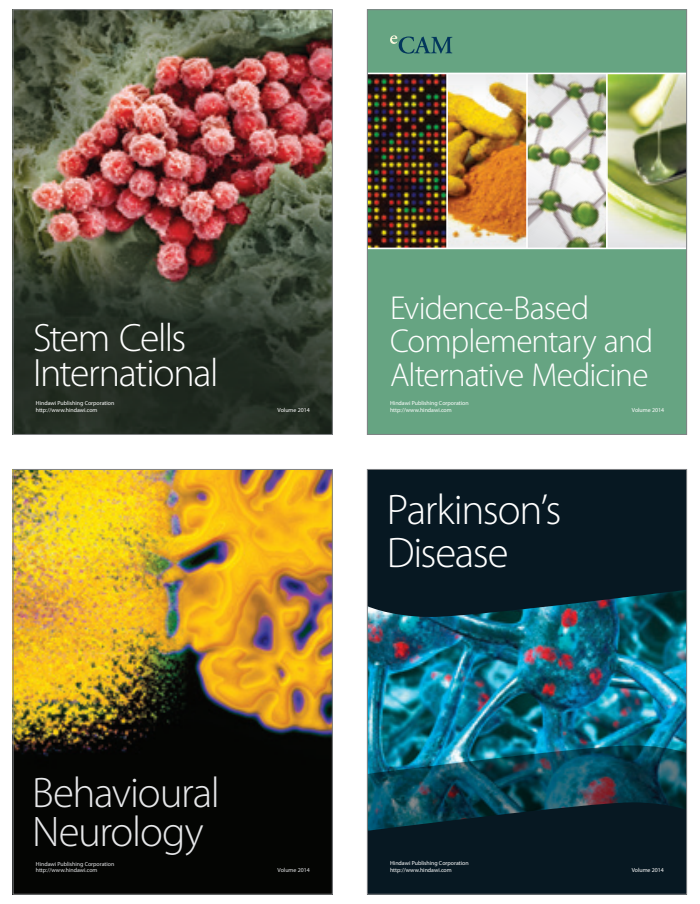
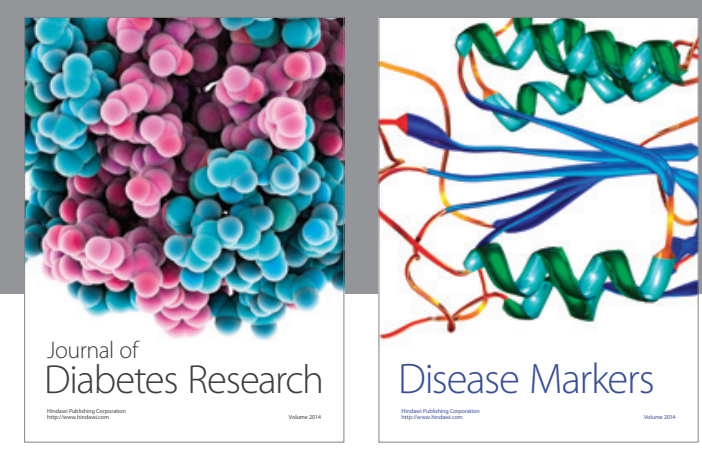

Disease Markers
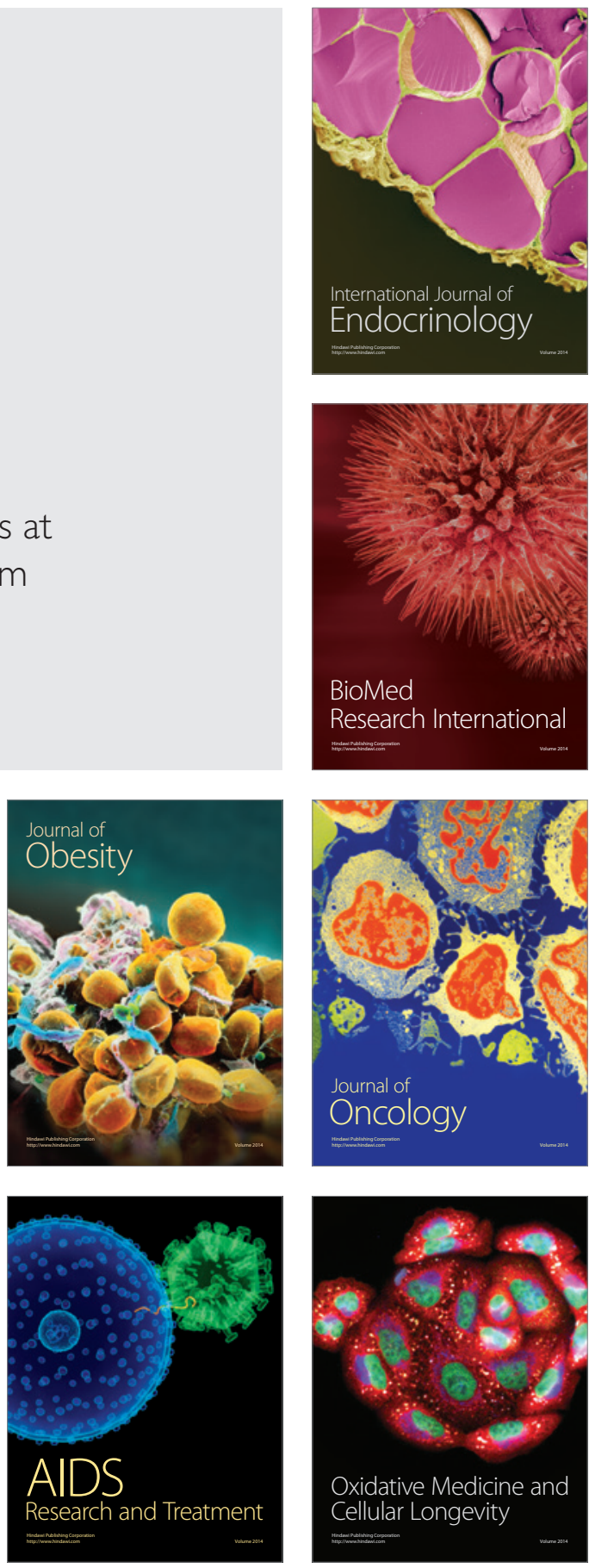